\title{
Relationship between tooth loss and mortality in 80-year-old Japanese community-dwelling subjects
}

Toshihiro Ansai ${ }^{*}$, Yutaka Takata², Inho Soh', Shuji Awano', Akihiro Yoshida', Kazuo Sonoki ${ }^{2}$, Tomoko Hamasaki ${ }^{3}$, Takehiro Torisu², Akira Sogame ${ }^{4}$, Naoko Shimada ${ }^{5}$, Tadamichi Takehara ${ }^{1}$

\begin{abstract}
Background: Findings from several studies suggest associations between tooth loss and health outcomes, including malnutrition, poor quality of life, and mortality, in older individuals. However, limited information is available regarding whether those associations remain true in very elderly subjects after adequately considering confounding factors such as sex and smoking status. Herein, we determined whether the number of teeth in 80 -year-old subjects is an independent predictor of mortality.

Methods: We initially contacted 1282 80-year-old community-dwelling individuals born in 1917, of whom 697 responded and participated in a baseline study, with follow-up examinations conducted 4 and 5.5 years later. Data from interviews and medical and oral examinations were obtained, and oral health was determined according to the number of teeth remaining in the oral cavity.

Results: A total of 108 and 157 subjects died in 4 years and 5.5 years, respectively, after the baseline study. Tooth loss was significantly associated with mortality at age 85.5 , but not at age 84 , after adjusting for potential confounders. When the analysis was stratified by sex, we found a stronger association in females in follow-up examinations conducted at both $4-$ and 5.5 years. On the other hand, the effect of tooth loss on mortality was not significantly different between smokers and non-smokers.
\end{abstract}

Conclusion: Tooth loss is a significant predictor of mortality independent of health factors, socio-economic status, and lifestyle in octogenarians, with a stronger association in females.

\section{Background}

Associations between tooth loss and mortality have been reported, though issues related to important confounding factors such as age, gender, and smoking status, which may be related to oral health and are also risk factors for mortality, remain to be clarified. In a number of studies, the age range of the subjects is broad [1-3], while there is a limited number of reports regarding the association of tooth loss and mortality in subjects who are the same age. An important merit of limiting a study population to the same age is that there is no need to consider the effects of age on changes that may potentially confound factors related to systemic condition. Thus, results

\footnotetext{
* Correspondence: ansai@kyu-dent.ac.jp

1 Division of Community Oral Health Science, Department of Health

Promotion, Kyushu Dental College, Kitakyushu, Japan
}

obtained with such a study design would be expected to reveal a much higher level of scientific evidence. In a search of published studies regarding the association between tooth loss and mortality in a single age group, we found 3 reports: a 10-year cohort study of 80-year-old subjects by Hämalainen et al., in which the hazard ratio (HR) for number of missing teeth was 1.026 [4], an 18 -year cohort study of 1803 70-year-old subjects by Österberg et al., in which the HRs for numbers of present teeth in males and females were 0.98 and 0.79 , respectively [5], and an investigation by Holm-Pedersen et al. of 573 70-year-old Danish individuals examined every 5 years for 20 years, in which the HR for mortality 21 years after beginning the study in subjects who were edentulous at the age 70 was 1.26 [6].
C Biomed Central

(c) 2010 Ansai et al; licensee BioMed Central Ltd. This is an Open Access article distributed under the terms of the Creative Commons Attribution License (http://creativecommons.org/licenses/by/2.0), which permits unrestricted use, distribution, and reproduction in any medium, provided the original work is properly cited. 
Smoking is a major risk factor for oral diseases, including periodontitis and systemic diseases, as well as cardiovascular disease and aspiration pneumonia. However, there is concern regarding the effects of smoking status, as previously published reports included inadequate measurement and control for smoking status, and confounding factors and effect modification have become increasingly important. Furthermore, another important issue is the source of study subjects, as nearly all published studies investigated subjects in Western countries. To the best of our knowledge, only 1 report focused on a single-age non-Western population, in which a Japanese population-based cohort was investigated [7]. In that study, the subjects were elderly (mean age approximately 82 years old) and the age range was narrow, though the sample size was small (59 males, 59 females) and smoking status was based on information obtained at the age of 60 .

In the present cohort study, we analyzed the association between number of teeth at the age of 80 -years old and mortality after 4 and 5.5 years, and then evaluated the effects of sex and smoking status on that association.

\section{Methods}

\section{Study population}

A total of 128280 -year-old candidates from 3 cities (Buzen, Yukuhashi, and Munakata), 4 towns (Katsuyama, Tsuiki, Toyotsu, and Kanda), 1 village (Shinyoshitomi), and 1 ward (Tobata of Kitakyushu City) in Fukuoka Prefecture on Kyushu Island in southern Japan were invited to participate. Those 9 locations were selected randomly from urban, suburban, and rural communities to achieve a balance of living environments in terms of socio-demographic backgrounds, dietary habits, health behaviors, and available medical care. This study was designed as an investigation of a representative population of individuals residing in the eastern area of Kyushu Island who were born in 1917. The percentage of 80-year-old individuals living in the study locations was approximately $0.62 \%$ of all residents, which was similar to the percentage $(0.64 \%)$ of 80 -yearold individuals residing in all of Kyushu. Of those 1282 individuals, 697 (54.4\%) (277 males, 420 females) agreed to participate in the present study and completed a questionnaire regarding lifestyle, and oral and systemic health, and also underwent physical, laboratory blood, and oral examinations. The Human Investigations Committee of Kyushu Dental College approved the survey and all subjects gave written informed consent prior to participation.

\section{Baseline data}

The baseline survey was performed in March 1998, during which time the participants took part in a face-to-face interview, and answered a questionnaire containing 37 questions about oral and systemic health status, use of medical (or dental) services, personal hygiene, healthcare practices (including smoking habit), and medical conditions [8]. Dental examinations were also performed using WHO criteria [9] by 3 trained dentists assisted by a dental nurse. In this study, only the number of teeth and denture status (fixed prostheses, partial or full dentures) were used for analysis. Remaining roots were not counted as retained teeth.

\section{Follow-up survey}

This study utilized data obtained from 1998 to 2003 and was designed to analyze the results of follow-up examinations of all subjects in our previous study at the ages of 84 and 85.5 years old. Information on the survival of the subjects was collected from the registers at the Public Health Centers of each district included in the study. Of all subjects who took part in the baseline survey, there were no losses during the follow-up period, as even subjects who had moved away from Fukuoka Prefecture after the initial survey were successfully traced.

\section{Statistical analyses}

Power analysis was performed using the software package G-power. The statistical power of this study was found to be $87 \%$, with sample sizes of 540 for $\mathrm{n} 1$ and 157 for $\mathrm{n} 2$, an effect size of 0.25 , and an $\alpha$ value of 0.05 set (two tailed t-test with accuracy mode), which demonstrated reasonable power. The main characteristics of participants who died versus those who remained alive during the follow-up period are reported as mean values (standard deviation, SD) or percentages. Differences between groups were tested using ANOVA for continuous variables and a Pearson chi-square test for categorical variables. Covariates determined at the baseline were sex, marital status, place of residence, medical history, laboratory blood test results (serum total cholesterol, fasting serum glucose, serum albumin), systolic and diastolic blood pressures, body mass index, regular physical activity, regular checkup by family doctor, selfrated general health, smoking habit, and daily alcohol consumption. Place of residence was included as a proxy for socioeconomic status.

The association between number of teeth and mortality was analyzed using multivariate logistic analysis. Preliminary analyses included univariate analyses of the association between number of teeth and mortality, covariates and number of teeth, and covariates and mortality. Multivariate analyses included variables that were related to number of teeth and mortality $(\mathrm{P}<0.05)$ in bivariate analyses, which included gender, smoking status, serum total cholesterol, fasting serum glucose, serum albumin, place of residence, marital status, and 
body mass index. We sequentially tested the effect modification of tooth loss-mortality associations by adding interaction terms between tooth loss and sex, and smoking status according to likelihood ratio tests.

All statistical analyses were performed using SPSS 14.0 for Windows (SPSS, Chicago, Illinois, USA).

\section{Results}

After 4 years, 108 of the subjects (58 males, 50 females) had died, while 157 (81 males, 76 females) had died by 5.5 years. Socio-demographic and medical characteristics of the study participants at the age of 80 are shown in Table 1 . The deceased individuals had lower total

Table 1 Sociodemographic and medical characteristics of 80-year-old subjects based on survival during 5.5-year follow-up period

\begin{tabular}{|c|c|c|c|}
\hline Characteristic & $\begin{array}{l}\text { Alive } \\
(n=540)\end{array}$ & $\begin{array}{l}\text { Died } \\
(n=157)\end{array}$ & $P$ value \\
\hline \multicolumn{4}{|l|}{ Sex } \\
\hline Female & $344(81.9 \%)$ & $76(18.1 \%)$ & 0.001 \\
\hline Male & $196(70.8 \%)$ & $81(29.2 \%)$ & \\
\hline \multicolumn{4}{|l|}{ Marital status ${ }^{a}$} \\
\hline Currently married & $253(74.2 \%)$ & $88(25.8 \%)$ & 0.022 \\
\hline Currently unmarried & $268(80.9 \%)$ & $63(19.1 \%)$ & \\
\hline \multicolumn{4}{|l|}{ Place of residence ${ }^{b}$} \\
\hline Ward & $149(85.6 \%)$ & $25(14.4 \%)$ & 0.008 \\
\hline City & $215(76.2 \%)$ & $67(23.8 \%)$ & \\
\hline Town/village & $170(73.3 \%)$ & $62(26.7 \%)$ & \\
\hline \multicolumn{4}{|l|}{ Medical history } \\
\hline CVD & $90(76.3 \%)$ & $28(23.7 \%)$ & 0.358 \\
\hline Cancer & $2(66.7 \%)$ & $1(33.3 \%)$ & 0.528 \\
\hline Pneumonia & $13(72.2 \%)$ & $5(27.7 \%)$ & 0.364 \\
\hline \multicolumn{4}{|l|}{ Medical examinations } \\
\hline Serum total cholesterol (mg/dL) & $208.1(37.4)$ & $196.8(40.1)$ & 0.002 \\
\hline Fasting serum glucose (mg/dL) & $116.5(44.5)$ & $138.9(74.6)$ & $<0.001$ \\
\hline Serum albumin $(\mathrm{g} / \mathrm{dL})$ & $4.3(0.29)$ & $4.1(0.35)$ & $<0.001$ \\
\hline Systolic blood pressure $(\mathrm{mmHg})$ & $150.8(23.6)$ & $149.2(21.8)$ & 0.463 \\
\hline Diastolic blood pressure $(\mathrm{mmHg})$ & $79.3(12.5)$ & $77.5(11.7)$ & 0.117 \\
\hline \multicolumn{4}{|l|}{ Disease risk factors } \\
\hline Body mass index & $22.9(3.3)$ & $21.9(3.2)$ & 0.002 \\
\hline \multicolumn{4}{|l|}{ Regular physical activity ${ }^{c}$} \\
\hline Yes & $300(77.9 \%)$ & $85(22.1 \%)$ & 0.614 \\
\hline No & $212(76.3 \%)$ & $66(23.7 \%)$ & \\
\hline \multicolumn{4}{|l|}{ Regular checkup by family doctor ${ }^{d}$} \\
\hline Yes & $430(77.5 \%)$ & $125(22.5 \%)$ & 0.688 \\
\hline No & $81(75.7 \%)$ & $26(24.3 \%)$ & \\
\hline \multicolumn{4}{|l|}{ Self-rated general healthe } \\
\hline Very good & $224(79.2 \%)$ & $59(20.8 \%)$ & 0.140 \\
\hline Good & $245(78.3 \%)$ & $68(21.7 \%)$ & \\
\hline Moderate & $61(69.3 \%)$ & $27(30.7 \%)$ & \\
\hline \multicolumn{4}{|l|}{ Smoking habit ${ }^{c}$} \\
\hline Smoker & $173(68.4 \%)$ & $80(31.6 \%)$ & $<0.001$ \\
\hline Non-smoker & $358(82.9 \%)$ & $74(17.1 \%)$ & \\
\hline \multicolumn{4}{|l|}{ Daily alcohol consumption ${ }^{f}$} \\
\hline Non-drinker & $221(75.4 \%)$ & $72(24.6 \%)$ & \\
\hline Drinker & $294(79.0 \%)$ & $78(21.0 \%)$ & 0.156 \\
\hline
\end{tabular}

Abbreviations: CVD, cardiovascular disease.

Data indicate the number of subjects (\%) or mean (SD).

Data available for ${ }^{\mathrm{a}} 672,{ }^{\mathrm{b}} 688,{ }^{\mathrm{c}} 663,{ }^{\mathrm{d}} 662,{ }^{\mathrm{e}} 684$, and ${ }^{\mathrm{f}} 665$ people, respectively.

Differences between groups were tested using a Pearson chi-square test for categorical variables and an ANOVA for continuous variables. 
Table 2 Dental characteristics of 80-year-old subjects based on survival during 5.5-year follow-up period

\begin{tabular}{|c|c|c|c|}
\hline Characteristic & $\begin{array}{c}\text { Alive } \\
(n=540)\end{array}$ & $\begin{array}{c}\text { Died } \\
(n=157)\end{array}$ & $P$ value \\
\hline Number of teeth ${ }^{a}$ & $8.4(9.0)$ & $6.7(8.2)$ & 0.031 \\
\hline Number of missing teeth ${ }^{a}$ & $23.5(9.0)$ & $25.3(8.2)$ & 0.026 \\
\hline Number of edentulous subjects ${ }^{a}$ & $178(75.4 \%)$ & $58(24.6 \%)$ & 0.196 \\
\hline Number of dentate subjects & $353(78.6 \%)$ & $96(21.4 \%)$ & \\
\hline \multicolumn{4}{|l|}{ Number of teeth \& denture status ${ }^{b}$} \\
\hline No denture & 89 (83.9\%) & 17 (16.1\%) & 0.108 \\
\hline PDs & $114(80.9 \%)$ & $27(19.1 \%)$ & \\
\hline Either PD or FD & $142(74.3 \%)$ & $49(25.7 \%)$ & \\
\hline FDs & $164(73.9 \%)$ & $58(26.1 \%)$ & \\
\hline \multicolumn{4}{|l|}{ Self-care ${ }^{c}$} \\
\hline Yes & $400(78.7 \%)$ & $108(21.3 \%)$ & 0.091 \\
\hline No & $103(72.1 \%)$ & $40(27.9 \%)$ & \\
\hline \multicolumn{4}{|l|}{ Toothbrushing $^{d}$} \\
\hline$\geq 2$ times/day & $240(83.6 \%)$ & $47(16.4 \%)$ & 0.004 \\
\hline$<2$ times/day & $213(73.7 \%)$ & $76(26.3 \%)$ & \\
\hline \multicolumn{4}{|l|}{ Regular checkups by dentist ${ }^{e}$} \\
\hline Yes & $360(79.6 \%)$ & $92(20.4 \%)$ & 0.027 \\
\hline No & 151 (71.9\%) & 59 (28.1\%) & \\
\hline
\end{tabular}

Abbreviations: FD, full denture; PD, partial denture (including fixed prostheses).

Data indicate the number of subjects (\%) or mean (SD).

Data available for ${ }^{\mathrm{a}} 685,{ }^{\mathrm{b}} 660,{ }^{\mathrm{c}} 651,{ }^{\mathrm{d}} 576$, and ${ }^{\mathrm{e}} 662$ people, respectively.

Differences between groups were tested using a Pearson chi-square test for categorical variables and an ANOVA for continuous variables.

cholesterol, higher glucose concentration, and lower serum albumin values as compared to those who remained alive, while BMI was higher in the survivors. Men and smokers were more likely to die during the follow-up period. The mortality rate was higher in males than in females at both 4 and 5.5 years, namely, 20.9\% vs. $11.9 \%$, and $29.2 \%$ vs. $18.1 \%$, respectively. The dental characteristics of the subjects are shown in Table 2 . Among the 697 subjects, the prevalence of edentulism was $33.9 \%(\mathrm{n}=236)$, and the proportion with 20 teeth or more was $15.2 \%(\mathrm{n}=106)$. As compared to edentulous males, edentulous females had a 1.3-fold higher rate of mortality in the 4-year follow-up examinations and a 1.2-fold higher rate of mortality in the 5.5-year follow-up examinations. The number of teeth (or number of missing teeth) and health-related behaviors such as brushing habit and regular check-ups by dentists differed between the survivors and deceased subjects.

Table 3 shows adjusted odds ratios (ORs) for number of teeth in regard to mortality at the 4-year follow-up examination. The overall OR was 0.980 [95\% confidence interval (CI), 0.953-1.007]. We performed interaction tests to investigate whether sex and smoking status were associated with tooth loss, and found a significant association between the risk of tooth loss and sex $(\mathrm{P}=$ 0.006), while there was no such association between smoking status and tooth loss. Accordingly, it was suggested that sex should be treated as an effect modifier in the following analyses. When stratified by sex, only the OR for number of teeth in females was significant (0.937, 95\% CI, 0.889-0.987), while there was not a significant association in males (OR, 1.004, 95\% CI, 0.969-1.040).

Table 4 shows adjusted ORs for number of teeth in regard to mortality at the 5.5-year follow-up examination. The overall OR for number of teeth was 0.972 (95\% CI, 0.948-0.996). Furthermore, the adjusted OR for female gender was 0.946 (95\% CI, 0.907-0.987), while that for male gender was 0.986 (95\% CI, 0.955-1.019), which was not significantly different.

\section{Discussion}

In the present longitudinal study, we investigated associations between tooth loss and mortality in 80-year-old Japanese subjects, and obtained the following important findings: (1) tooth loss was associated with higher mortality in our 80-year-old subjects; (2) the associations differed due to sex, with female gender significantly associated with mortality; and (3) smoking status did not affect the association between tooth loss and mortality. Abnet et al. [1] reported a difference between genders for the association between tooth loss and mortality, as that association was stronger in males, though the difference between females and males was only slight. Also, 2 different reports presented by Japanese researchers found an association between the number of

Table 3 Odds ratios for effect of number of teeth on mortality at 4-year follow-up examination according to sex: Multivariate logistic regression analysis

\begin{tabular}{|c|c|c|c|c|c|c|}
\hline & \multirow[b]{2}{*}{ Adjusted OR $(95 \% \mathrm{Cl})^{a}$} & \multirow[b]{2}{*}{$\mathbf{P}$} & \multicolumn{4}{|c|}{ OR by sex } \\
\hline & & & & Cases & Adjusted OR $(95 \% \mathrm{Cl})^{\mathrm{b}}$ & $\mathbf{P}$ \\
\hline \multirow[t]{2}{*}{ Number of teeth } & $0.980(0.953-1.007)$ & 0.148 & Males & 277 & $1.004(0.969-1.040)$ & 0.841 \\
\hline & & & Females & 420 & 0.937 (0.889-0.987) & 0.014 \\
\hline
\end{tabular}

OR: odds ratio, $\mathrm{Cl}$ : confidence interval.

${ }^{a}$ Adjusted for sex, smoking habit, serum total cholesterol, fasting serum glucose, serum albumin, place of residence, marital status, and body mass index.

${ }^{b}$ Adjusted for smoking habit, serum total cholesterol, fasting serum glucose, serum albumin, place of residence, marital status, and body mass index.

'Stratified by sex due to results of interaction tests, which indicated a statistically significant difference for risk related to tooth loss between males and females $(P=0.006)$ (see statistical analyses). 
Table 4 Odds ratios for effect of number of teeth on mortality at 5.5-year follow-up examination according to sex: Multivariate logistic regression analysis

\begin{tabular}{|c|c|c|c|c|c|c|}
\hline & \multirow[b]{2}{*}{ Adjusted OR $(95 \% \mathrm{Cl})^{\mathrm{a}}$} & \multirow[b]{2}{*}{$\mathbf{P}$} & \multicolumn{4}{|c|}{ OR by sex ${ }^{c}$} \\
\hline & & & & Cases & Adjusted OR $(95 \% \mathrm{Cl})^{\mathrm{b}}$ & $\mathbf{P}$ \\
\hline \multirow[t]{2}{*}{ Number of teeth } & $0.972(0.948-0.996)$ & 0.023 & Males & 277 & 0.986 (0.955-1.019) & 0.399 \\
\hline & & & Females & 420 & $0.946(0.907-0.987)$ & 0.011 \\
\hline
\end{tabular}

OR: odds ratio, $\mathrm{Cl}$ : confidence interval.

${ }^{a}$ Adjusted for sex, smoking habit, serum total cholesterol, fasting serum glucose, serum albumin, place of residence, marital status, and body mass index.

${ }^{b}$ Adjusted for smoking habit, serum total cholesterol, fasting serum glucose, serum albumin, place of residence, marital status, and body mass index.

'Stratified by sex due to results of interaction tests, which indicated a statistically significant difference for risk related to tooth loss between males and females $(P=0.006)$ (see statistical analyses).

teeth and mortality in elderly males [2,7]. Their findings did not coincide with ours, though the reasons for the differences remain unclear. They might be partly explained by the different age range and life-style related factors of the study subjects.

There are a number of reports regarding the association between number of teeth and mortality. However, some of those analyzed subjects with a broad age range, used a small number of subjects, studied mixed genders, and/or investigated subjects with smoking habit information lacking. In a literature search, we found only 3 reports of a cohort study of the association between number of teeth and mortality in a single age elderly population [4-6]. The baseline ages of the subjects in those reports were either 80 or 70 years old, and they presented similar conclusions stating that tooth loss is independently associated with mortality and independent of other potential confounding factors. On the other hand, treatment of smoking habit varied among those 3 reports, as Hämalainen et al. [4] did not provide adequate information regarding smoking status, while the others indicated smoking status, but did not show the effects of smoking [5,6]. An explanation for our result regarding the role of smoking status may be related to survivor effects. In our subjects who survived to the age of 85.5 , the effect of smoking may have been reduced.

The mechanism of the association between tooth loss and mortality has been hypothesized to consist of 2 pathways; an infection and inflammation pathway, and a nutritional pathway, as reported by Janket et al. [10]. Tooth loss is known to mainly be caused by periodontal disease, the most common oral infectious disease, indicating an inflammatory burden from a past infection, and periodontitis is associated with a steady increase in circulatory levels of pro-inflammatory cytokines $[11,12]$. In addition, inadequate dentition due to tooth loss may affect eating behavior, including mastication and food choice, causing individuals to substantially reduce their intake of fruits, vegetables, and other key nutrients [13], which have been shown to be associated with increased survival and lower cardiovascular mortality [14].
Furthermore, adults who have no natural teeth and wear complete replacement dentures tend to ingest a high fat, low fiber diet [15]. However, we have no direct evidence to support any of those speculations at the present time.

A limitation of our study is that the sample consisted largely of generally healthy elderly subjects, who might have been more eager and/or able to participate. Thus, our findings may indicate the association in generally healthy elderly subjects. The response rate to participate in the study was $54 \%$, which was related to location and health-oriented behavior, as individuals who resided in towns, and those who regularly attended check-up examinations by a family doctor or dentist had higher response rates. On the other hand, several findings have been presented regarding the effect of inadequate dental status on mortality of institutionalized elderly individuals [16], in which poor dentition was found to be associated with high overall mortality, thus systematic attention to dental status is recommended.

\section{Conclusions}

In this study, our results showed a significant relationship between tooth loss and mortality in 80-year-old individuals, even after extensive adjustment for potential confounding variables. Since a stronger association was found in females, we recommend that researchers include gender when performing related statistical analyses. Our findings also have significant implications in terms of public health strategy, because they indicate that improving oral health and keeping more natural teeth will increase the health status of the population as well as longevity.

\section{Acknowledgements}

The authors extend their gratitude to the subjects who participated in this investigation. This investigation was supported by a research grant from the 8020 Promotion Foundation, and by Grants-in-Aid (B) 15390655 and (B) 17390566 from the Ministry of Education, Culture, Sports, and Technology of Japan.

\section{Author details}

'Division of Community Oral Health Science, Department of Health

Promotion, Kyushu Dental College, Kitakyushu, Japan. ${ }^{2}$ Division of General Internal Medicine, Department of Health Promotion, Kyushu Dental College, 
Kitakyushu, Japan. ${ }^{3}$ Division of Nutrition, Department of Home Economics, Kyushu Women's University, Kitakyushu, Japan. ${ }^{4}$ Kurate Office for Health, Human Services and Environmental Issues, Nogata, Japan. ${ }^{5}$ Kitakyushu Public Health and Welfare Bureau, Kyushu Dental College, Kitakyushu, Japan.

\section{Authors' contributions}

The epidemiological study was supervised by $\Pi T$ and TA. TA, YT, IS, SA, AY, $\mathrm{KS}, T H, T$, AS, NS, and TT participated in the epidemiological study. TT initially designed the study. TA contributed toward analysis and interpretation of data, and wrote the first draft of the manuscript. All authors read and approved the final version of the report.

\section{Competing interests}

The authors declare that they have no competing interests.

Received: 18 August 2009 Accepted: 1 July 2010 Published: 1 July 2010

\section{References}

1. Abnet CC, Qiao YL, Dawsey SM, Dong ZW, Taylor PR, Mark SD: Tooth loss is associated with increased risk of total death and death from upper gastrointestinal cancer, heart disease, and stroke in a Chinese population-based cohort. Int J Epidemiol 2005, 34:467-74.

2. Fukai $K$, Takiguchi T, Ando $Y$, Aoyama H, Miyakawa $Y$, Ito G, Inoue M, Sasaki H: Mortality rates of community-residing adults with and without dentures. Geriatr Gerontol Int 2008, 8:152-9.

3. Heitmann BL, Gamborg M: Remaining teeth, cardiovascular morbidity and death among adult Danes. Prev Med 2008, 47:156-60.

4. Hämalainen $\mathrm{P}$, Meurman JH, Keskinen $\mathrm{M}$, Heikkinen E: Relationship between dental health and 10-year mortality in a cohort of communitydwelling elderly people. Eur J Oral Sci 2003, 111:291-6.

5. Österberg T, Carlsson GE, Sundh V, Mellstrom D: Number of teeth-a predictor of mortality in 70-year-old subjects. Community Dent Oral Epidemiol 2008, 36:258-68.

6. Holm-Pedersen P, Schultz-Larsen K, Christiansen N, Avlund K: Tooth loss and subsequent disability and mortality in old age. J Am Geriatr Soc 2008, 56:429-35.

7. Morita I, Nakagaki H, Kato K, Murakami T, Tsuboi S, Hayashizaki J, Toyama A, Hashimoto M, Simozato T, Morishita N, et al: Relationship between survival rates and numbers of natural teeth in an elderly Japanese population. Gerodontology 2006, 23:214-8.

8. Ansai T, Takata Y, Soh I, Akifusa S, Sogame A, Shimada N, Yoshida A, Hamasaki T, Awano S, Fukuhara M, et al: Relationship between chewing ability and 4-year mortality in a cohort of 80-year-old Japanese people. Oral Dis 2007, 13:214-9.

9. WHO: Oral Health Surveys. Geneva: WHO, 3 1987, 22-44.

10. Janket $S J$, Qvarnstrom $M$, Meurman JH, Baird $A E$, Nuutinen $P$, Jones JA: Asymptotic dental score and prevalent coronary heart disease. Circulation 2004, 109:1095-100.

11. D'Aiuto F, Parker M, Andreou G, et al: Periodontitis and systemic inflammation: control of the local infection is associated with a reduction in serum inflammatory markers. J Dent Res 2004, 83:156-160.

12. Montebugnoli L, Servidio D, Miaton RA, Prati C, Tricoci P, Melloni C: Poor oral health is associated with coronary heart disease and elevated systemic inflammatory and haemostatic factors. J Clin Periodontol 2004, 31:25-29.

13. Sheiham A, Steele J: Does the condition of the mouth and teeth affect the ability to eat certain foods, nutrient and dietary intake and nutritional status amongst older people? Public Health Nutr 2001, 4:797-803.

14. Tucker KL, Hallfrisch J, Qiao N, Muller D, Andres R, Fleg JL: The combination of high fruit and vegetable and low saturated fat intakes is more protective against mortality in aging men than is either alone: the Baltimore Longituginal Study of Aging. J Nutr 2005, 135:556-561.

15. Allen PF: Association between diet, social resources and oral health related quality of life in edentulous patients. J Oral Rehabil 2005, 32:623-628.

16. Ohrui T, Matsui T, Yoshida M, Yoneyama T, Adachi M, Akagawa Y, He M, Yamaga M, Arai H, Sasaki H, et al: Dental status and mortality in institutionalized elderly people. Geriatr Gerontol Int 2006, 6:101-108.

\section{Pre-publication history}

The pre-publication history for this paper can be accessed here: http://www.biomedcentral.com/1471-2458/10/386/prepub

doi:10.1186/1471-2458-10-386

Cite this article as: Ansai et al:: Relationship between tooth loss and mortality in 80-year-old Japanese community-dwelling subjects. BMC Public Health 2010 10:386.

\section{Submit your next manuscript to BioMed Central and take full advantage of:}

- Convenient online submission

- Thorough peer review

- No space constraints or color figure charges

- Immediate publication on acceptance

- Inclusion in PubMed, CAS, Scopus and Google Scholar

- Research which is freely available for redistribution

Submit your manuscript at www.biomedcentral.com/submit 